\title{
Hysteroscopic Surgery as an Alternative for Hysterectomy
}

\author{
Chang-Sheng Yin ${ }^{1}$ and Fung-Wei Chang ${ }^{2}$ \\ ${ }^{1}$ China Medical University Taipei Branch, Vice-Superintendent, \\ National Defense Medical Center, \\ ${ }^{2}$ Reproductive Medicine Center, \\ Department of Obstetric and Gynecology, \\ Tri-Service General Hospital, Taipei, \\ Taiwan
}

\section{Introduction}

Abnormal uterine bleeding (AUB) is a common complaint in reproductive age women. The World Health Organization reported that in developing countries, 8-27\% of women suffered heavy menstrual bleeding. (1) Hysterectomy remains the gold-standard treatment, but can be associated with significant morbidity. In the past gynecologists will be criticized for performing hysterectomies for women with AUB but without any uterine pathology. Over the past two decades, several non-invasive or minimally invasive procedures have emerged as alternative treatment modalities. But do these new treatments remain effective over time? In the present chapter, I discuss first generation hysteroscopic ablation techniques (EA), as an alternative to hysterectomy, that have been extensively studied over the last 20 years but are actually underused. Because a great number of valuable papers and books have already been published on this topic, for the sake of simplicity references have been kept to a minimum. Also I will share my personal experiences as a hysteroscopist who has performed more than 1000 cases of operative hysteroscopic surgery in Taiwan over the last 15 years.

\section{Indications for hysterectomy}

A hysterectomy is an operation to remove a woman's uterus. Sometimes, the ovaries and fallopian tubes are also removed. Hysterectomies are very common - one in three women in the United States has had one by age 60.

The following benign diseases are the most common reasons for hysterectomy:

- Abnormal uterine bleeding (AUB) that persists despite treatment

- Uterine leiomyomata (fibroids)

- Adenomyosis not cured by medicine or conservative surgery

- Uterine prolapse - when the uterus drops into the vagina

- Chronic pelvic pain; intractable to medical treatments 
Before a patient undergoes a hysterectomy, it is important to discuss alternative treatments with the patient. After hysterectomy her periods will stop, and she will no longer be able to get pregnant. If both ovaries are removed, a patient will enter menopause. Although hysterectomy is one of the most commonly used gynecological procedures, in recent years the number of patients undergoing hysterectomy has decreased in some area of the world. (2)

The possible reasons for this could be the availability of new emerging minimally invasive alternative procedures and the adverse effects after hysterectomy. $(2,3)$ Current 'save the uterus ' attitude in the world has also become a support to narrow the range of indications of hysterectomy. Hysterectomy can alleviate all menstrual-related clinical symptoms, including dysmenorrhea and premenstrual symptoms, and the overall patient satisfaction rate with this approach is approximately $80 \%$. However, it also has some disadvantages such as frequent peri-operative complications, prolonged operating time, delayed recovery, and high cost. Recent studies have shown that women below 50 years of age who undergo hysterectomy with or without oophorectomy are at a significantly higher risk of all-cause mortality and coronary heart diseases. $(3,4)$ Traditionally, hysterectomy has also been known to have a major psychological impact. Over the past 15-20 years, several noninvasive or minimally invasive procedures have emerged as alternative treatment modalities. Currently available alternative treatment modalities include insertion of levonorgestrel-intrauterine system (LNG-IUS), myomectomy, myolysis, uterine artery embolization, magnetic resonance imaging-focused ultrasonography, hysteroscopic endometrial ablation (EA), and second-generation global endometrial ablation.

\section{Alternatives for hysterectomy}

Avoiding unnecessary hysterectomy is a challenge. Abnormal uterine bleeding (AUB), defined as excessive or irregular bleeding, occurs in about $10-30 \%$ of women of reproductive age. Uterine leiomyomata (fibroids) is the most common benign gynecological tumor, occurring in about $30 \%$ of women. The 2002 report from Organization for Economic Co-operation and Development showed that rates ranged from a low of 114 per 100000 women in Mexico to a high of 526 in Norway. In Taiwan, the incidence of hysterectomy was approximately 268 to 303 per100 000 women from 1996 to 2001. Fibroids are the most common indication for hysterectomy in Taiwan; a total of 22,000 hysterectomies are performed each year. (5) The overall cost of treating fibroids in US was estimated at \$2.1 billion in 2000 . More than $70 \%$ of those costs were directly related to hysterectomy.

Asymptomatic uterine leiomyomata may not require treatment, but in about $20-80 \%$ of cases, leiomyomata is accompanied by AUB and pelvic symptoms. AUB and leiomyomata are the main indications for hysterectomy. If AUB can be successfully treated with alternative modalities, then the need for hysterectomy may be reduced.

Recently, several non-invasive or minimally invasive procedures have emerged as alternative treatment modalities include insertion of levonorgestrel-intrauterine system (LNG-IUS), myomectomy, myolysis, uterine artery embolization, magnetic resonance imaging-focused Ultrasonography. While hysteroscopic endometrial ablation, and secondgeneration global endometrial ablation (EA), are to be choiced when menorrhagia is the primary indication and endometrial anatomy is appropriate. For patients requiring interventional treatment, selection among these procedures depends on the patient's age, symptoms, coexisting conditions, and reproductive plans. 
EA is a suitable alternative for the treatment of AUB. Resectoscopic techniques are performed under hysteroscopic visualization, using resectoscopic instruments to ablate the endometrium or remove submucosal leiomyomata. Hysteroscopic EA and myomectomy have become increasingly popular treatment modalities for women with AUB. From 2003 to 2006 , in England, EA was performed in $60 \%$ of cases of surgically treated AUB. (6)

The 2009 Cochrane Database meta-analysis of 21 randomized trials regarding resectoscopic ablation showed the following results: (a) the amenorrhea rate was $38 \%$ at 1 year and $48 \%$ at 2-5 years, (b) the patient satisfaction rate was $88 \%$ at 1 year and $87 \%$ at $2-5$ years, and (c) the subsequent surgery rate was $25 \%$ for any surgery (including hysterectomy) and $19 \%$ for hysterectomy alone. (7) Since 1996, I have performed more than 1000 hysteroscopic EAs at 3 hospitals in Taiwan. The results were comparable to those in other medical literature, but had a lower subsequent surgery rate of approximately $5 \% .(8,9)$ In my recent study, I followed up 334 women who were eligible for a follow-up after surgery; the follow-up duration ranged from 7 months to 109 months (mean, 48.7 months). Of the 334 women, 161 (48\%) had normal flow, 33 (10\%) had hypomenorrhea, 31 (9\%) had heavy flow, and $82(25 \%)$ continued to experience amenorrhea. Seventeen (5\%) women were not satisfied with the results, and $7(2 \%)$ underwent hysterectomy because of EA failure. Ten women (3\%) underwent EA again. The subsequent surgery rate in my series was 5\%. (8)

Although this procedure needs training and skills, it is less expansive and underused in many countries. Many insurance policies such as Taiwan's National Health Insurance do not cover second-generation endometrial ablation.

Most clinicians still perform hysterectomy as the first choice in treatment of women presenting intractable AUB.

The history and evolution of hysteroscopic endometrial ablation: In 1805, Bozzini described his simple instrument used to inspect the urethra of a living human but later was banned and censured for "undue curiosity."

In 1869, Pantaleoni took the first look inside the uterine cavity using a cystoscope to observe and cauterize uterine polyps in a 60 -year-old woman.

In 1879, the first "modern" hysteroscope was designed and produced by Nitze by incorporating an interior light source with an optical system permitting magnification.

In 1925, Rubin introduced uterine distention with carbon dioxide .

The rod lens system designed by Harold H.Hopkins, (10) together with the fiber optic light transmission invented by Fourestiere in 1952, (11) has allowed extraordinary advances in operative hysteroscopy.

In 1869, Pantaleoni reported the first successful hysteroscopy (using cystoscopy), when he described a 60-year-old woman with postmenopausal bleeding and found an intrauterine polyp and cauterized it with silver nitrate. This was not only the first diagnostic hysteroscopy but also the first demonstration of intrauterine surgery.

Modern day operative hysteroscopy relies on two developments: the glass fiber optic for illumination and the uterine distention, and the hysteroscopic endometrial ablation 
developed from the occurrence of Asherman's syndrome that completes destruction of the endometrium will cause amenorrhea.

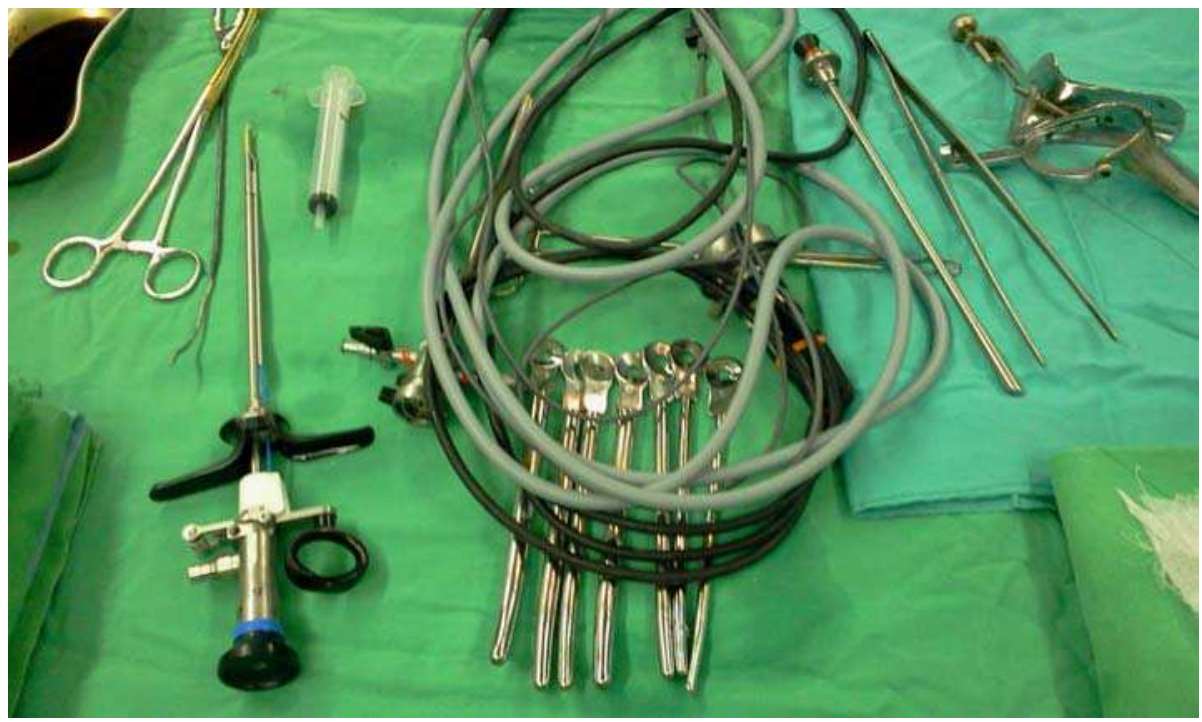

Fig. 1. instrumentation for hysteroscopic endometrial ablation include rigid resectoscope, cables, speculum, and dilators.

In 1976, Neuwirth \& Amin used the urologic resectoscope to assist in the removal of a pedunculated submucous leimyomas in 5 cases. Two years later (1978) Neuwirth used a new hysteroscopic technique with a cutting loop and infusion distention fluid ( $32 \%$ dextran 70) system.

In 1979 Goldrath first performed hysteroscopic endometrial ablation by using the Nd:YAG laser.

In 1981 he reported on the successful treatment of intractable uterine bleeding in 21 of 22 cases.

\section{The general assessment and management of AUB}

The FIGO PALM-COEIN classification for abnormal uterine bleeding (AUB):

In 2011 a new classification system for abnormal uterine bleeding (AUB) was approved by the FIGO Executive Board. (12) AUB, can be classified as acute, chronic, intermenstrual or intermittent forms, which represents a common clinical problem facing the gynecologist.

Acute AUB was defined as an episode of heavy bleeding that is of sufficient quantity to require immediate intervention to prevent further blood loss.

Chronic AUB be defined as bleeding from the uterine corpus that is abnormal in volume, regularity, and/or timing, and has been present for the past 6 months. Chronic AUB would not require immediate intervention. 
Intermenstrual or intermediate bleeding (IMB) occurs between clearly defined cyclic and predictable menses. Such bleeding may occur at random times or may manifest in a predictable fashion at the same day in each cycle.

The FIGO group recommended the word "metrorrhagia," and dysfunctional bleeding (DUB) should be abandoned.

Acute AUB may present in the context of existing chronic AUB or might occur without such a history. Although women of reproductive age with acute AUB require immediate intervention, their follow-up may be largely dependent upon whether they require investigation and ongoing care for an underlying chronic condition.

The prevalence of AUB, the difficulties in identifying its causes, and the cost of management argue for reliable diagnostic techniques and treatment strategies. An ideal approach would promptly identify patients with organic disease, facilitate patient comprehension and choice of treatment options, improve treatment efficacy, and reduce overall cost of care.

FIGO differentiates 9 categories and are arranged according to an acronym (PALM-COEIN) shown as below:

AUB-P: Polyps in the endometrium that are diagnosed by ultrasound or hysteroscopy. This classification system does not further categorize polyps on the basis of size, location, or number.

AUB-A: Adenomyosis which can be most accurately diagnosed on the basis of tissue analysis of a hysterectomy specimen, but in everyday clinical practice, MRI and ultrasound are both used to establish the diagnosis.

AUB-L: Leiomyomas, which can be solitary or multiple, can be located close to the cavity (submucosal), in the myometrium (intramural), close to the outer surface (subserosal), or independent of the uterus (parasitic). On the basis of these characteristics, further subcategories have been created.

AUB-M: Malignancy, including hyperplasia and endometrial cancer. The diagnosis requires the histologic analysis of a biopsy sample.

AUB-C: Coagulopathy, which in most cases is diagnosed in adolescents. Most abnormalities are the result of von Willebrand disease.

AUB-O: Ovulatory dysfunction, in which cycle length and volume of flow are unpredictable.

AUB-E: Endometrial problems, typically related to abnormal prostaglandin synthesis, but which could be the result of infection as well.

AUB-I: Iatrogenic causes, the result of exogenous hormone administration or anticoagulant therapy.

AUB-N: Nonclassified causes such as arteriovenous malformations and myometrial hypertrophy. Women with both acute and chronic AUB should be evaluated for anemia. In the absence of any other identifiable source, the clinician would proceed in a systematic fashion, designing the assessment to address each of the components of the FIGO classification system. 
Current treatment for AUB is recommended only when symptoms such as pain, severe anemia, or hydronephrosis due to ureteral obstruction are present and unacceptable to patients. There is no evidence that women with no symptoms or with mild symptoms benefit from intervention. Medical therapy such as acetaminophen and non-steroidal antiinflammatory drugs (NSAIDs) for pain relief is useful in some patients, but does not reduce uterine bleeding. A variety of hormonal therapies, mifepristone, and gonadotropin-releasing hormone agonists and antagonists have limited effects in reducing uterine volume and bleeding, but most of these treatments have not been evaluated in randomized trials. $(11,12)$ Patients with ovulatory dysfunction ( AUB-O) are best treated with oral contraceptives, NSAIDs (antiprostaglandins), or progestins or progestin-released IUD. Cyclic use of progestins and oral contraceptives reduce AUB, but ergot derivatives do not.

For patients requiring surgical alternatives, the current principal options include myomectomy, endometrial ablation, uterine fibroid embolization, and magnetic resonanceguided focused ultrasound (MR-g FUS). A thoughtful discussion of the options with patients is essential in choosing the most appropriate treatment for patients. Selection among these procedures depends on the patient's age, symptoms, and their preference.

A large study from the United Kingdom in 2005 observed that 16,100 out of 37,298 hysterectomies were performed as a result of AUB.

Wade et al. suggested that for women with AUB who were older than 40 years and did not desire pregnancy, the best treatment trial was with oral contraceptive pills (OCPs), followed by second generation endometrial ablation as the most cost-effective strategy. Unfortunately, currently in Taiwan the second generation endometrial ablation therapy is not covered by Taiwan National Health Insurance. Therefore, my personal experience and the experience of many other investigators have demonstrated that hysteroscopic endometrial ablation in patients with AUB is a reasonable alternative to classic hysterectomy. The advantages include no abdominal incision, less discomfort, very brief recovery period, no hysterectomy complications, fewer psychological problems and lower cost. Endometrial ablation is the hysteroscopic alternative to hysterectomy as treatment for women with AUB.

The pre-operative investigation procedure for women with AUB:

It is advised to begin a work-up when a patient complaining of AUB with a pictorial blood assessment chart scores (PBACS) above 150 or the patient's quality of life changes. A detailed clinical history and complete physical examination may reveal blood coagulation disorders, liver and renal disease. Laboratory studies include complete blood count (CBC), and monitoring of TSH, prolactin, and androgen levels if there are signs and symptoms of hypothyroidism, galactorrhea, hirsutism or acne. The gynecological examination with bimanual examination must be performed with specific attention given to the existence of possible lesions in the cervix, uterus, adnexa, urethra, bladder and rectum. Spe cial attention for the presence of endometrial cancer is also mandatory. It is mandatory to perform an office endometrial biopsy in all patients contemplating hysteroscopy surgery. A determination of uterine size is also useful because patients with uterine cavities greater than $10 \mathrm{~cm}$ in length can expect less than optimal results.

Endometrial sampling directed by hysteroscopy may increase the accuracy in evaluating patients with abnormal intrauterine bleeding. Ultrasound and or saline infusion sonography 
(SIS) may detect the presence of intrauterine lesions. The abdominal and or vaginal ultrasound evaluation of the pelvic organs is also very helpful for operation.

\section{The procedure of the hysteroscopic endometrial ablation}

The operation is always performed under spinal anesthesia in the proliferative phase of the menstrual cycle; if not, then a pre-operative thorough 3-min suction curettage is done.

Souter et al. conducted a meta-analysis about pre-operative administration of agents producing endometrial atrophy before surgery, and suggest it is associated with shortened operation times, greater rate of amenorrhea, and reduced dysmenorrhea. I prefer to pretreat the endometrium by trying to time the procedure in the early proliferative phase and using the suction curette to remove the rest of the endometrium.

Informed consent is obtained before the operation. Misoprostol (Cytotec) 200mcg \# x2 per vagina is given the night before operation to ripen the cervix.

The patient is placed in the dorsal lithotomy position. A simultaneous laparoscopy is not performed.

The vulva area, vagina and cervix are cleaned with disinfecting solution. A bimanual examination should be performed to determine whether the position of the uterus is anteverted or retroverted. After placing a single valve speculum in the vagina, the cervix is grasped with a single-toothed tenaculum which allows for traction on the cervix. The cervix is then dilated to Hegar No:10,

A continuous fluid rigid hystero-resectoscope equipped with a 30 degree fore-oblique hysteroscope is slowly without force introduced into the uterine cavity. If there is any difficulty during insertion of the resectoscope, there is a risk of uterine perforation.

The $5 \%$ dextrose is an isotonic solution with sufficient optical quality, no electric conductivity, no allergic potential, and is the cheapest and most readily available in $500 \mathrm{ml}$ bottle form. It is safe for endometrial ablation but the danger of fluid overload and pulmonary edema still exists. The uterine cavity is distended with a solution of $5 \%$ dextrose under a gravity feed system with a 2-litre-bottle connected to the resectoscope by urologic tubing. Uterine distension is created by raising the bags to a suitable height, usually 1.0-1.5 $\mathrm{m}$ above the uterine cavity. Inspection of the input and output fluid volume is done every 5 minutes by the circulating nurse during the operation. The negative balance is limited to $2000 \mathrm{ml}$. After running distention fluid for about one minute, a clear field to see both uterine cornu and fundus is considered as the most important indicator that the operation can begin.

I prefer partial ablation to treat the posterior wall endometrium (depth $<3 \mathrm{~mm}$ ), initially by the cutting loop electrode with the current set at $120 \mathrm{~W}$ of cutting current. From my right hand side to left, both lateral side of the endometrium are resected and ablated down next to the isthmus. The rest of the endometrium is then coagulated using the rollerball electrode, with the current set at $60 \mathrm{~W}$ of coagulation current.

The current total or global EA procedures cause significant intrauterine adhesion and contractures, and are associated with long-term adverse effects, which include painful 
central hematometra, cornual hematometra, and postablation tubal sterilization syndrome in patients with tubal ligation. Postablation tubal sterilization syndrome consists of painful hematosalinges, resulting from active endometrial tissue near the cornual region and endometrial scarring and contractures that prevent menstrual egress. A partial endometrial ablation procedure in which only one (anterior or posterior) wall is resected and ablated was proposed to prevent intrauterine scarring and contractures, and thus avoid such complications $(16,17)$. This approach allows the normal endometrial surface to oppose the injured exposed myometrial surface, so that adhesions and contractures do not occur. To date, there has been only one report of a pregnancy after partial EA. This pregnancy only proceeded to 28 weeks of gestation, and does not yet show potential benefits for women wishing to retain fertility (18).

After that any bleeding is coagulated using the roller ball electrode at a power of 40-60 W of coagulation current.

For women in whom uterine polyps or myomas are found, concomitant resections are done. Submucosal myoma resections are carried out using unipolar cutting-loop resection techniques.

All of the resected endometrial strips, myomatous fragments and polyps are sent for histological evaluation.

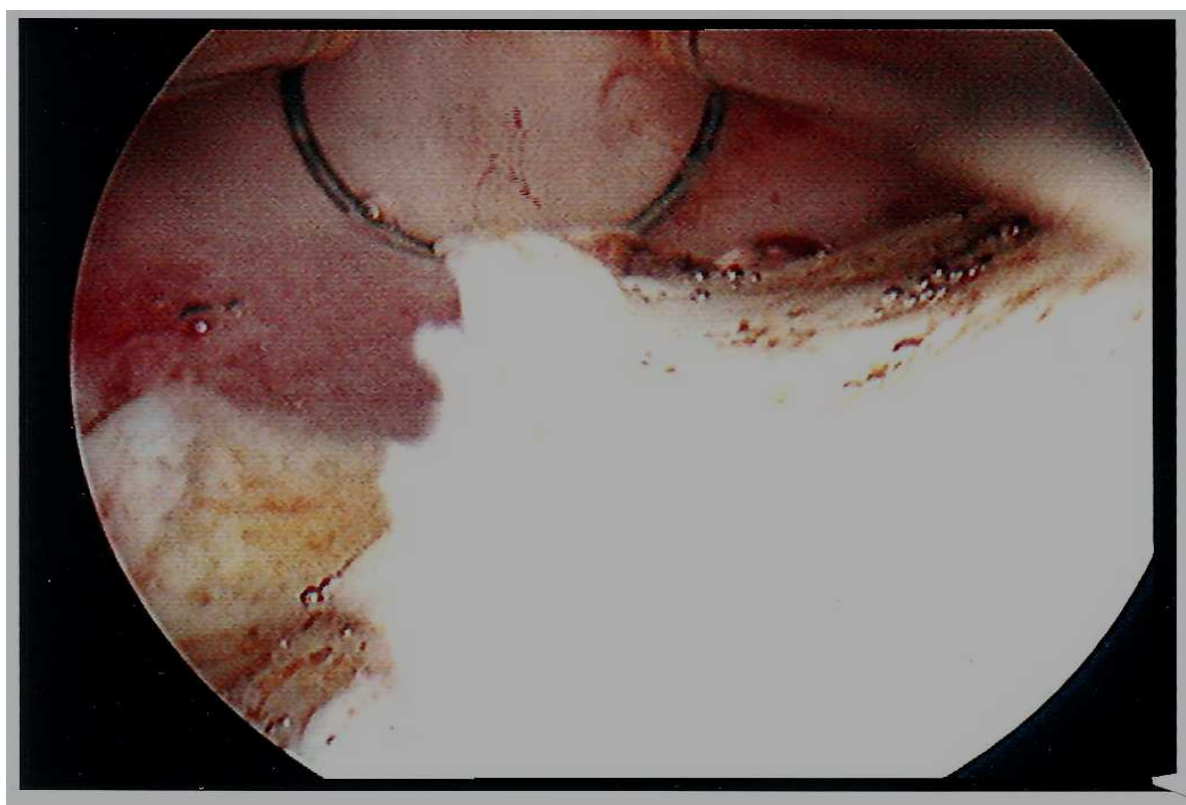

Fig. 2. Placement of the loop electrode behind this $4 \mathrm{~cm}$ submucosal myoma in a 34 year old woman under direct vision

Most patients after endometrial resection will have a fairly light period for a few days, then become a watery pink discharge last for a week or two to three weeks. 
The first post-operative office visit is scheduled for 1 week later, and 1 month after surgery, then followed up every 6 months by telephone, mail or at clinics.

Patient satisfaction is also important to assess the question "How satisfied or dissatisfied are you with the result of the surgery related to menstrual flow and dysmenorrhea?" Patients rate their overall satisfaction on a 4 -points scale( $1=$ very satisfied, $2=$ satisfied, $3=$ dissatisfied, $4=$ very dissatisfied). The need for repeat surgery or hysterectomy is also recorded. Patients with post-operative AUB may receive medical treatment, or if AUB, and dysmenorrhea are persistent, hysterectomy may be needed.

\section{The safety of the procedure}

Electrosurgical endometrial resection is not as easy as you think when watching an expert performing it. It is actually associated with more complications than any other procedure in the whole field of endoscopic surgery and performed only by obstetrician-gynecologists after thorough training. The most serious complications are fluid overload resulting in pulmonary edema and electrolytes imbalances. The first signs of pulmonary edema may perceived by the drop in oxygen saturation. Early and rapid treatment of furosemide and positive pressure ventilation may have a complete recovery. I used Dextrose $5 \%$ in water as the distention fluid because of its effective low-viscosity and electrolytically non-conductive and cheap. The most important factors predisposing to dilutional hyponatremia were nulliparity, type of preoperative endometrial preparation,increased uterine size and cavity length, concurrent myomectomy and duration of surgery. Fluid monitoring with input and output recording is essential during all operative hysteroscopic cases.

Uterine perforation may occur while removal of intrauterine chips, and can cause serious internal organs and vascular injury if unnoticed during resection.

There are several tips to avoiding uterine perforation complications ;

1. Learn the physics associated with electrosurgery and the hydrodynamics inherent in the fluid systems used.

2. Electrical energy should never be activated unless the surgeon can clearly see the operating field.

3. The roller-ball or wire loop must always be pulled towards the operator and never activated when it is pushed away from the surgeon. Most important is to avoid perforation; it can be surprisingly difficult to detect.

4. Always keep uterine "perforation" in mind, especially in cases with distorted uterine cavity and never activate the radiofrequency energy when bleeding blurs the field, as damage can occur to a wide variety of vessels and organs: the most dangerous complication is uterine perforation with electrical thermal injury and trauma.

Trauma to major blood vessels: aorta, inferior vena cava, mesenteric artery, sacral artery, iliac artery.

Trauma to bowel resulting in peritonitis and/or septicemia.

Trauma to bladder and/or ureter requiring laparotomy or ureteric stents.

Technique troubleshooting; 
Poor vision may be due to bleeding and debris and bubbles.

\section{Solution;}

Bleeding - first make sure the instrument is in the uterine cavity then increase distention pressure and coagulate the bleeder.

Debris and bubbles - withdraw the scope running the distention fluid and rinse the perforations in the outer shaft.

- Hemorrhage requiring balloon tamponade, laparotomy or hysterectomy or blood loss leading to death.

- Distention medium overload.

- $\quad$ Other rare complications such as unintended pregnancy.

- Intrauterine adhesions and hematometra, burns.

Nearly all of my procedures have been completed safely; however, two women sustained uterine perforation and underwent hysterectomies. In a third woman, the procedure was abandoned because of suspected endometrial malignancy. None of my patients have showed any signs or symptoms of fluid overload or hyponatremia.

In my series over a 15-year-period, six pregnancies occurred after the procedure, and the mean age of these women was 36 years (range, 34-40 years). Because pregnancies have been reported after endometrial ablation, patients cannot be guaranteed that this is a sterilization procedure, and the risk potential for a subsequent pregnancy must be explained and sterilization or contraception offered. Tubal ligation does not reduce distention media absorption and therefore need not be a prerequisite.

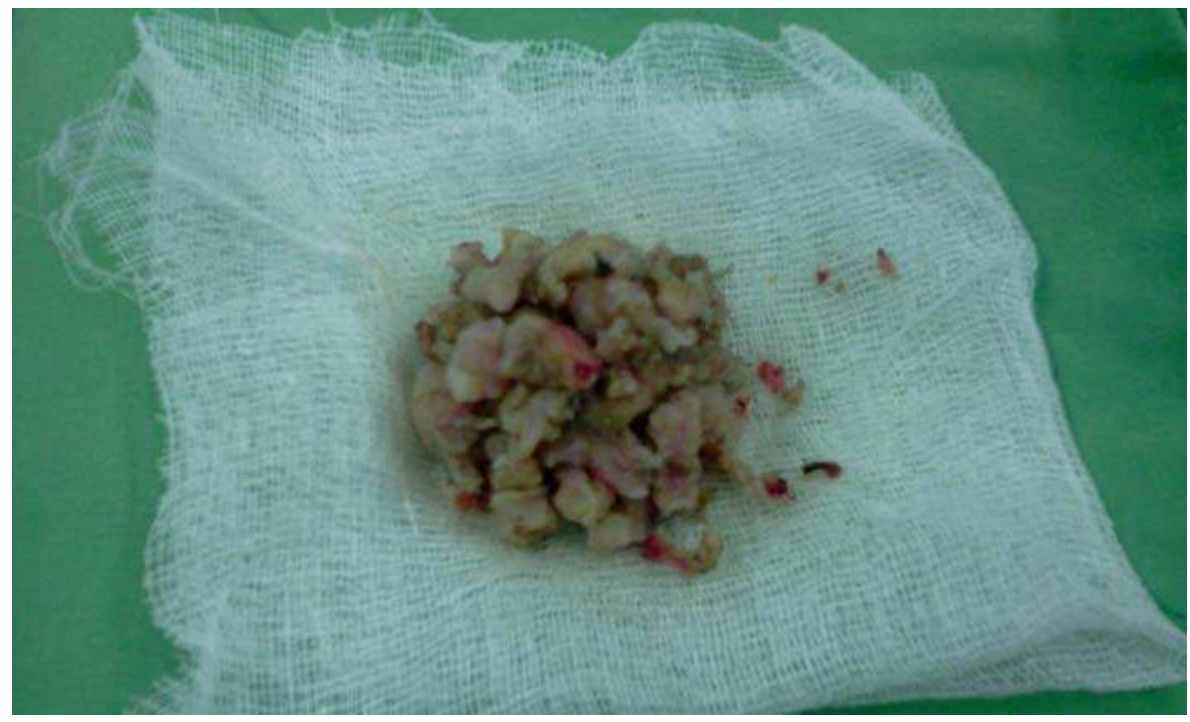

Fig. 3. Collection of myoma chips after resection.

Is endometrial ablation really decreased the hysterectomy rate? 
An earlier report from England suggested that the increasing use of endometrial ablation did not reduce the rate of hysterectomy . (19) Despite initial optimism in the United States, two studies showed that the hysterectomy rate remained unchanged at 5.68 per thousand women between 1988 and 1997. (20,21) According to a 2010 population-based NHI database in Taiwan, demonstrated that there have been considerable changes in the surgical types of hysterectomy performed in Taiwan during the last 10 years, but the annual numbers of hysterectomies remained stationary. (5) This means that hysteroscopic endometrial ablation and other alternatives to hysterecotomy are still underused in Taiwan and many parts of the world. Many factors might influence the selection of treatment for uterine and menstrual disorders, some are surgeon, and others are patient-related. Widespread critical clinical skills and more educations about the alternatives to hysterectomy for surgeons and patients are needed.

\section{Conclusion}

Hysteroscopic endometrial ablation is the logic alternative choice for women with AUB after failed medical treatment.

Although hysteroscopic endometrial ablation offers many advantages, it is actually underused by gynecologist in the world. Every gynecologist must to learn the skill of diagnostic and operative hysteroscopy as their everyday routine practice. Even newer developments in $2^{\text {nd }}$ generation global endometrial ablation, every gynecologist should retain their hysteroscopic operative skills whenever need.

\section{References}

[1] Harlow SD, Campbell OM. Epidemiology of menstrual disorders in developing countries: A systematic review. BJOG 2004;111:6-16.

[2] Babalola EO, Bharucha AE, Schleck CD, et al. Decreasing utilization of hysterectomy: a population-based study in Olmsted County, Minnesota, 1965-2002. Am J Obstet Gynecol. 2007;196:214.e1.

[3] Ingelsson E, Lundholm C, Johansson AL, et al. Hysterectomy and risk of cardiovascular disease: a population based cohort study. Eur Heart J. 2010 Dec 24. [Epub ahead of print].

[4] Parker WH, Broder MS, Chang E, et al. Ovarian conservation at the time of hysterectomy and long-term health outcomes in the nurses' health study. Obstet Gynecol. 2009;113:1027-1037.

[5] Wu MP, Haung KH, Long CY. et al. Trends in Various Types of Surgery for Hysterectomy and Distribution by Patient Age, Surgeon Age, and Hospital Accreditation: 10-Year Population-Based Study in Taiwan. Analysis on the causes and effects of hysterectomy in Taiwan. J Minim Invasive Gynecol. 2010, 17, 612-619

[6] Cromwell DA, Mahmood TA, Templeton A, et al. Surgery for menorrhagia within English regions: variation in rates of endometrial ablation and hysterectomy. BJOG. 2009;116:1373.

[7] Lethaby A, Hickey M, Garry R, et al. Endometrial resection/ablation techniques for heavy menstrual bleeding. Cochrane Database Syst Rev 2009; :CD001501. 
[8] Yin CS, Wei RY, Chao TC, et al. Hysteroscopic endometrial ablation without endometrial preparation. Int J Gynaecol Obstet. 1998;62(2):167-72.

[9] Yin CS. Pregnancy after hysteroscopic endometrial ablation without endometrial preparation: A report of five cases and a literature review. Taiwan J Obstet Gynecol. 2010;49(3):311-319.

[10] Hamou JE, Historical background. In: Hamou J, ed. Hysteroscopy and microcolpolhysteroscopy. Text and Atlas. East Norwalk, CT: Appleton and Lange,1991:1-11.

[11] Goldrath MH, Fuller TA, Segal S. Laser photovaporization of endometrium for treatment of menorrhagia. Am J Obstet Gynecol 1981; 140:14-19. Bibliographic Links Library Holdings .

[12] FIGO classification system (PALM-COEIN) for causes of abnormal uterine bleeding in nongravid women of reproductive age. Malcolm G. Munroa, b, , , Hilary O.D. Critchleyc, Michael S. Broderd, Ian S. Frasere and for the FIGO Working Group on Menstrual Disorders. Int J Obstet Gynecol, vol.113, no.1 p3-13, April 2011

[13] Parker WH. Uterine myomas: management. Fertil Steril. 2007;88:255-71.

[14] Stewart EA. Uterine fibroids. Lancet 2001;357:293-8.

[15] Kjerulff KH, Erickson BA, Langenberg PW. Chronic gynecological conditions reported by US women: findings from the National Health Interview Survey, 1984 to 1992. Am J Public Health 1996;86:195-199.

[16] McCausland AM, McCausland VM. Partial rollerball endometrial ablation: a modification of total ablation totreat menorrhagia without causing complications from intrauterine adhesions. Am J Obstet Gynecol 1999;180:1512-21.

[17] McCausland AM, McCausland VM. Frequency of symptomatic corneal hematometra and postablation tubal sterilization syndrome after total rollerball endometrial ablation: a 10-year follow-up. Am J Obstet Gynecol 2002;186:1274-84.

[18] Maouris P. Pregnancy after planned partial endometrial resection. Aust N Z J Obstet Gynaecol 1994;34:122-3.

[19] Bridgeman SA. Trends in endometrial ablation and hysterectomy for dysfunctional uterine bleeding in the Mersey region. Gynecol Endosc. 1996;5:5-8.

[20] Wilcox LS, Koorin LS, Pokras R, Strauss LT, Xia Z, Petersen HB. Hysterectomy in the United States 1988-1990. Obstet Gynecol. 1994;83:549-555.

[21] Farquhar Steiner CA. Hysterectomy rates in the United States, 1990- 1997. Obstet Gynecol. 2002;99:229-234. 


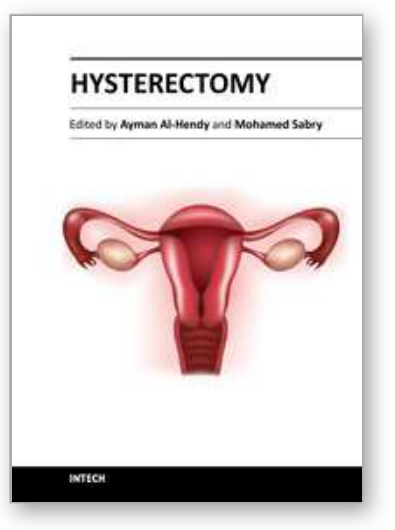

\author{
Hysterectomy \\ Edited by Dr. Ayman Al-Hendy
}

ISBN 978-953-51-0434-6

Hard cover, 426 pages

Publisher InTech

Published online 20, April, 2012

Published in print edition April, 2012

This book is intended for the general and family practitioners, as well as for gynecologists, specialists in gynecological surgery, general surgeons, urologists and all other surgical specialists that perform procedures in or around the female pelvis, in addition to intensives and all other specialities and health care professionals who care for women before, during or after hysterectomy. The aim of this book is to review the recent achievements of the research community regarding the field of gynecologic surgery and hysterectomy as well as highlight future directions and where this field is heading. While no single volume can adequately cover the diversity of issues and facets in relation to such a common and important procedure such as hysterectomy, this book will attempt to address the pivotal topics especially in regards to safety, risk management as well as pre- and post-operative care.

\title{
How to reference
}

In order to correctly reference this scholarly work, feel free to copy and paste the following:

Chang-Sheng Yin and Fung-Wei Chang (2012). Hysteroscopic Surgery as an Alternative for Hysterectomy, Hysterectomy, Dr. Ayman Al-Hendy (Ed.), ISBN: 978-953-51-0434-6, InTech, Available from: http://www.intechopen.com/books/hysterectomy/hysteroscopic-surgery-as-an-alterative-treatment-modalities

\section{INTECH}

open science | open minds

\section{InTech Europe}

University Campus STeP Ri

Slavka Krautzeka 83/A

51000 Rijeka, Croatia

Phone: +385 (51) 770447

Fax: +385 (51) 686166

www.intechopen.com

\section{InTech China}

Unit 405, Office Block, Hotel Equatorial Shanghai

No.65, Yan An Road (West), Shanghai, 200040, China

中国上海市延安西路65号上海国际贵都大饭店办公楼405单元

Phone: +86-21-62489820

Fax: +86-21-62489821 
(C) 2012 The Author(s). Licensee IntechOpen. This is an open access article distributed under the terms of the Creative Commons Attribution 3.0 License, which permits unrestricted use, distribution, and reproduction in any medium, provided the original work is properly cited. 